\title{
Conocimiento de la técnica de automonitoreo de glucosa en pacientes diabéticos en un municipio del Estado de Hidalgo
}

Knowledge of the glucose self-monitoring technique in diabetic patients in a municipality of Hidalgo State.

\author{
Margarita Lazcano-Ortiz ${ }^{a}$, Paloma M Balderas-Sierra ${ }^{b}$, Brenda Godinez-Jiménez ${ }^{b}, M$ \\ Fernanda Monroy-Vargas ${ }^{b}$, Margarita Pérez-Gómez ${ }^{b}$, M Carmen Ramírez-Salazar $^{b}$, Reyna \\ C. Jiménez-Sánchez ${ }^{c}$, María L. Sánchez-Padilla ${ }^{d}$
}

\begin{abstract}
:
Introduction: There are several types of diabetes depending on their cause. People with diabetes may have elevations or decreases in levels unexpectedly. It is necessary to monitor glucose levels, to detect and prevent the occurrence of complications.

Objective: To evaluate the knowledge of the glucose self-monitoring technique in patients with Type 2 Diabetes Mellitus.

Material and method: Non-experimental, transactional, descriptive research. A sample of 30 older adults with Type 2 Diabetes Mellitus, non-probabilistic sampling for convenience. A validated instrument "Knowledge of the self-monitoring technique in patients with Type 2 Diabetes Mellitus" was applied, by author Fogel Gerardo, with 22 items, informed consent was delivered. Results: The average age was 61 years ( $\mathrm{SD}=9,290 ; 45-88)$, with a female prevalence of $67 \%$, Housewife occupation of $60 \%$ and a primary schooling level of $37 \%$. It was identified that $100 \%$ of the sample has its own glucometer, as for the knowledge of the selfmonitoring technique, it was observed that $60 \%$ perform their self-monitoring in the morning and that $70 \%$ do it once a day. The $40 \%$ have the right knowledge about normal glucose values, while the other $60 \%$ have a wrong knowledge. Conclusions: There is a lack of knowledge about the self-monitoring technique in a large part of diabetic patients of the Health Center of the Municipality of Arenal, Hidalgo; since little more than half fail to identify basic steps for proper self-monitoring technique.
\end{abstract}

Keywords:

Knowledge, self-monitoring, Diabetes Mellitus type 2.

\begin{abstract}
Resumen:
Introducción: Existen varios tipos de diabetes dependiendo de su causa. Las personas con diabetes pueden tener elevaciones 0 disminuciones de los niveles de manera inesperada. Es necesario monitorear los niveles de glucosa, para detectar y prevenir la aparición de complicaciones. Objetivo: Evaluar el conocimiento de la técnica de automonitoreo de glucosa en pacientes con Diabetes Mellitus tipo 2. Material y método: Investigación de tipo no experimental, transaccional, descriptivo. Una muestra de 30 adultos mayores con Diabetes Mellitus tipo 2, muestreo no probabilístico por conveniencia. Se aplicó un instrumento validado "Conocimiento de la técnica de automonitoreo en pacientes con Diabetes Mellitus tipo 2", del autor Fogel Gerardo, con 22 ítems, se entregó consentimiento informado. Resultados: El promedio de edad fue de 61 años ( $D E=9.290 ; 45-88$ ), con una prevalencia del sexo femenino del 67\%, de ocupación Ama de Casa del $60 \%$ y un grado de escolaridad primaria de $37 \%$. Se identificó que el $100 \%$ de la muestra cuenta con glucómetro propio, en cuanto al conocimiento de la técnica de automonitoreo, se observó que el $60 \%$ realiza su automonitoreo por la mañana y que el $70 \%$ lo realizan una vez al día. El $40 \%$ tienen el conocimiento correcto sobre los valores normales de glucosa, mientras que el otro $60 \%$ tienen un conocimiento erróneo Conclusiones: Existe la falta de conocimiento sobre la técnica de automonitoreo en gran parte de los pacientes diabéticos del Centro de Salud del Municipio del Arenal, Hidalgo; ya que poco más de la mitad no llegan a identificar pasos básicos para una adecuada técnica de automonitoreo.
\end{abstract}

\section{Palabras Clave:}

Conocimiento, Automonitoreo, Diabetes Mellitus tipo 2

\footnotetext{
a Autor de Correspondencia, Profesor Investigador, SNI, Universidad Autónoma del Estado de Hidalgo, Instituto de Ciencias de la Salud, Área académica de Enfermería. ORCID: 0000-0002-9653-9291. Pachuca Hgo. México, Email: margarita_lazcano@uaeh.edu.mx

Alumnos de Licenciatura en Enfermería de la Universidad Autónoma del Estado de Hidalgo, Instituto de Ciencias de la Salud, Área académica de Enfermería, Pachuca Hgo. México. Email: margarita_lazcano@uaeh.edu.mx

${ }^{c}$ Profesor investigador Universidad Autónoma del Estado de Hidalgo, Instituto de Ciencias de la Salud, Área académica de Enfermería, Pachuca Hgo. México. Email: cristyji@ hotmail.com

${ }^{\text {d }}$ Profesor investigador Universidad Autónoma del Estado de Hidalgo, Instituto de Ciencias de la Salud, Área académica de Enfermería, Pachuca Hgo. México. Email: marialuisasanchezpadilla@yahoo.com.mx
} 


\section{Introducción}

La diabetes es una enfermedad crónica que aparece cuando el páncreas no produce insulina suficiente o cuando el organismo no utiliza eficazmente la insulina que produce. Existen varios tipos de diabetes dependiendo de su causa, sin embargo, en todos los tipos existe la posibilidad de tener los niveles de glucosa alterados $^{[1]}$

El automonitoreo es un elemento esencial para lograr las metas de control de los niveles de glucosa. La educación para el autocuidado de la diabetes es un elemento crítico dentro de la atención a las personas con diabetes y aquellos sujetos con riesgo de desarrollar la enfermedad; el autocuidado es considerado uno de los principales componentes del complejo tratamiento que la persona con Diabetes Mellitus tipo 2 debe asumir, exigiendo que tenga conocimiento y habilidades para desarrollar los comportamientos de autocuidado que son esenciales para el tratamiento y mantener la calidad del control metabólico, reduciendo las morbidades asociadas a las complicaciones de la Diabetes Mellitus tipo 2.(DM2).

En ese sentido, la educación para el autocuidado es recomendada por la Organización Mundial de Salud por ser una herramienta que hace a la persona con DM2 protagonista de su tratamiento, permitiendo mayor adhesión al esquema terapéutico y, así, prevención de las complicaciones originadas por ese problema crónico. En enfermería es de gran importancia el uso del glucómetro, ya que es necesario que los pacientes tengan conocimiento acerca del auto monitoreo para generar en ellos autonomía con el fin de llevar un buen control de la enfermedad y prevenir o retrasar complicaciones.

La DM2, es el tipo más común de la diabetes, es la primera causa de mortalidad general en México, esta aumenta el riesgo de enfermedades cardiovasculares.

De ahí que la educación del paciente, de manera específica a través de la enseñanza, es un componente integral en la atención de enfermería, donde es posible cuidar enseñando ${ }^{[2]}$.

\section{Marco Conceptual}

La diabetes mellitus (DM) comprende un grupo de trastornos metabólicos frecuentes que comparten el fenotipo de la hiperglucemia. Existen varios tipos diferentes de DM resultado de una interacción compleja entre genética y factores ambientales. De acuerdo con la causa de la DM, los factores que contribuyen a la hiperglucemia pueden ser deficiencia de la secreción de insulina, disminución de la utilización de glucosa o aumento de la producción de ésta ${ }^{[3]}$.

Los principales factores de riesgo para la DM2 son los siguientes:

- Los antecedentes de diabetes en padres 0 hermanos.

- La obesidad, definida por presentar al menos un $20 \%$ de peso corporal mayor del deseado o por tener un índice de masa corporal de al menos 27 $\mathrm{kg} / \mathrm{m} 2$.

- La inactividad física.

- La raza/la etnia.

- En mujeres un antecedente de DM gestacional, de síndrome de ovario poliquístico, o el parto de un bebé con un peso superior a $4 \mathrm{~kg}$.

- La hipertensión arterial (>130/85 en adultos), un colesterol HDL >35 mg/dL y/o una concentración de triglicéridos $>250 \mathrm{mg} / \mathrm{dL}$.

- Síndrome metabólico.

- La persona con DM del tipo 2 tiene un inicio lento de las manifestaciones y a menudo no es consciente de la enfermedad hasta que requiere asistencia sanitaria para algún otro problema. La hiperglucemia en el tipo 2 no suele ser tan grave como en el tipo 1, pero los síntomas son similares, especialmente la poliuria y la polidipsia. La polifagia no suele observarse a menudo, y la pérdida de peso es infrecuente. 
- Otras manifestaciones son también resultado de la hiperglucemia: visión borrosa, fatiga, parestesias e infecciones cutáneas. Si la insulina disponible disminuye, especialmente en períodos de estrés físico o emocional, la persona con DM del tipo 2 puede desarrollar cetoacidosis diabética (CAD), pero esta situación es poco frecuente ${ }^{[4]}$.

\section{Complicaciones agudas:}

- Hiperglucemia

- El fenómeno del amanecer

- El fenómeno de Somogyi

- Estado hiperglucémico hiperosmolar.

- Cetoacidosis diabética.

- Hipoglucemia

\section{Complicaciones crónicas:}

- Alteraciones del sistema cardiovascular

- Enfermedad coronaria

- Hipertensión

- Accidente cerebrovascular

- Enfermedad vascular periférica

- Retinopatía diabética

- Nefropatía diabética ${ }^{[4]}$.

\section{Teoría del Déficit de Autocuidado}

Dorothea E. Orem presenta su teoría del déficit de autocuidado como una teoría general compuesta por tres teorías relacionadas entre sí: Teoría de autocuidado, teoría del déficit autocuidado y la teoría de los sistemas de Enfermería ${ }^{[5,6]}$.

Esta teoría ofrece a los profesionales de la enfermería herramientas para una atención de calidad, en cualquier situación relacionada con el binomio salud-enfermedad tanto a personas enfermas, como personas que están tomando decisiones sobre su salud y personas sana que desean mantenerse o modificar conductas de riesgo para su salud.

El autocuidado es una función humana reguladora que debe aplicar cada individuo de forma deliberada con el fin de mantener su vida y su estado de salud, desarrollo y bienestar, por tanto es un sistema de acción. La elaboración de los conceptos de autocuidado, necesidad de autocuidado, y actividad de autocuidado conforman los fundamentos que permiten entender las necesidades y las limitaciones de acción de las personas que pueden beneficiarse de la enfermería. Como función reguladora del hombre, el autocuidado es diferente de otros tipos de regulación del funcionamiento y el desarrollo humano ${ }^{[6]}$.

El autocuidado debe aprenderse y aplicarse de forma deliberada y continúa en el tiempo, siempre en correspondencia con las necesidades de regulación que tienen los individuos en sus etapas de crecimiento y desarrollo, estados de salud, características sanitarias o fases de desarrollos específicas, factores del entorno y niveles de consumo de energía ${ }^{[7]}$.

La teoría establece los requisitos de autocuidado, que además de ser un componente principal del modelo forma parte de la valoración del paciente, el término requisito es utilizado en la teoría y es definido como la actividad que el individuo debe realizar para cuidar de sí mismo, Dorotea E Orem propone a este respecto tres tipo de requisitos:

1. Requisito de autocuidado universal.

2. Requisito de autocuidado del desarrollo

3. Requisito de autocuidado de desviación de la salud.

Los requisitos de autocuidado de desviación de la salud, son la razón u objetivo de las acciones de autocuidado que realiza el paciente con incapacidad o enfermedad crónica $^{[8]}$.

Para lograr mejores niveles de satisfacción en este ámbito, es necesario la observación y evaluación constante de la calidad de los servicios de enfermería, da la medida de la importancia de establecer pautas o sistemas que refuercen científicamente el trabajo de la enfermera sobre el déficit de autocuidado. La glucemia nos hace referencia a la presencia de moléculas de glucosa en sangre. En otras palabras, la concentración de glucosa en el plasma sanguíneo, medida en mg/dL. 
La automonitorización de la glucemia capilar (AGC) es una herramienta clave en el tratamiento actual de la diabetes, tanto para el paciente como para el equipo médico. Su objetivo es obtener la información necesaria de los niveles de glucosa en sangre en diversos momentos del día para así poder ajustar el plan de tratamiento alimentario, la actividad física y el tratamiento farmacológico, especialmente las dosis de insulina ${ }^{[9]}$.

El glucómetro o medidor de glucosa en sangre capilar se utiliza para medir la cantidad de glucosa que hay en una gota de sangre. Permite saber si los niveles de glucosa del niño están dentro de su franja ideal. Hay diferentes tipos de medidores ${ }^{[10]}$. .

\section{Medidores de glucosa y las tiras reactivas para la diabetes}

El uso de un glucómetro para vigilar y controlar el nivel de azúcar en la sangre es parte de la vida diaria de millones de personas con diabetes. ${ }^{[11]}$. Los medidores de glucosa y las tiras reactivas son dispositivos médicos controlados por la Administración de Alimentos y Medicamentos de los Estados Unido (FDA, por sus siglas en inglés). ${ }^{[11]}$.

La codificación es primordial para prevenir serias complicaciones potenciales en la salud asociadas con errores en la dosis de insulina. Los valores generados por el glucómetro, son usados por los pacientes para ajustar su dieta, ejercicio y dosis de insulina. Si el glucómetro no está bien programado, no dará un resultado fiable. Si este paso no es realizado correctamente, el glucómetro puede dar resultados erróneos que llevaran a una mala corrección de insulina. Para que un glucómetro realice su labor correctamente, hace falta realizar el proceso de codificación. Este proceso se realiza cada vez que se use una nueva caja de tiras reactivas; para que el glucómetro reconozca las nuevas tiras reactivas, (cada caja de tiras reactiva tiene su propio código).

\section{Cuidados del glucómetro: calibración y limpieza}

Se necesita un resultado fiable de glucemia capilar para poder tomar una decisión que involucre la salud de las personas. La calibración del glucómetro es una parte esencial del autoanálisis de glucemia capilar, ya que un glucómetro mal calibrado lleva a resultados erróneos. Hay muchas razones por las cuales un glucómetro puede estar descalibrado, y ante cualquier duda sobre el resultado, debemos calibrarlo[10].

La limpieza del glucómetro es una parte básica del cuidado del dispositivo. Si el dispositivo está sucio puede ofrecer un mal resultado que puede llevar a una complicación en el manejo y tratamiento de la enfermedad. Así como un diabético debe cuidar su nutrición, mantener una vida activa y saludable, cuidar sus pies; así también debe cuidar su glucómetro y prestarle la atención necesaria.

El autoanálisis de la glucemia capilar es un componente imprescindible para el autocontrol de la diabetes, que a su vez constituye una parte fundamental del tratamiento integral de la misma. El autocontrol ha demostrado sus efectos beneficiosos sobre el control metabólico de la enfermedad, expresado como HbA1c, así como su capacidad para predecir las hipoglucemias y para que el paciente tome mayor conciencia de su enfermedad ${ }^{[10]}$. .

\section{Objetivo.}

Identificar el nivel de conocimiento que el paciente diabético tiene sobre la técnica de automonitoreo de la glucosa y su enfermedad.

\section{Material y método}

Investigación de tipo no experimental, transaccional, descriptivo. Una muestra de 30 adultos mayores con Diabetes Mellitus tipo 2, muestreo no probabilístico por conveniencia, a través de una encuesta de recopilación verbal, por medio de preguntas cerradas de opción 
múltiple. Se aplicó un instrumento validado con el nombre de "Conocimiento de la técnica de automonitoreo en pacientes con Diabetes Mellitus tipo 2", del autor Fogel Gerardo, con 22 ítems, se entregó consentimiento informado.

\section{Resultados}

En la muestra estudiada se encontró un promedio de edad de 61 años, (DE=9.290; 45-88), con predominio del sexo femenino del $67 \%$, la ocupación que mayor resulto fue Ama de Casa $60 \%$ y el grado de escolaridad primaria con 37\%. Se observó que el $40 \%$ de los pacientes tienen el conocimiento correcto sobre los valores normales de glucosa, mientras que el otro $60 \%$ tienen un conocimiento erróneo, se identificó que solo el $23 \%$ de los pacientes conocen correctamente que significa la codificación del glucómetro, mientras que el otro $77 \%$ desconoce a qué se refiere la palabra codificación, en cuanto a la técnica de automonitoreo se observó que el $80 \%$ de los pacientes si desinfectan con alcohol antes de realizar la punción mientras que el resto no, y que solo el $50 \%$ saben la zona de punción correcta.

Figura 1 Años con la enfermedad

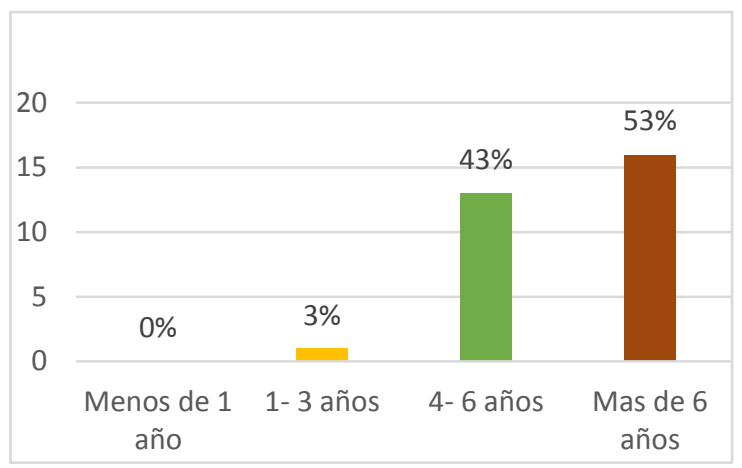

Se observa en la figura 1 que poco más de la mitad de pacientes del centro de salud del Municipio del Arenal llevan más de 6 años con Diabetes Mellitus 2, y que tan solo el $3 \%$ de pacientes tiene de 1 a 3 años con la enfermedad.
Figura 2 Conocimiento de valores normales de Glucosa

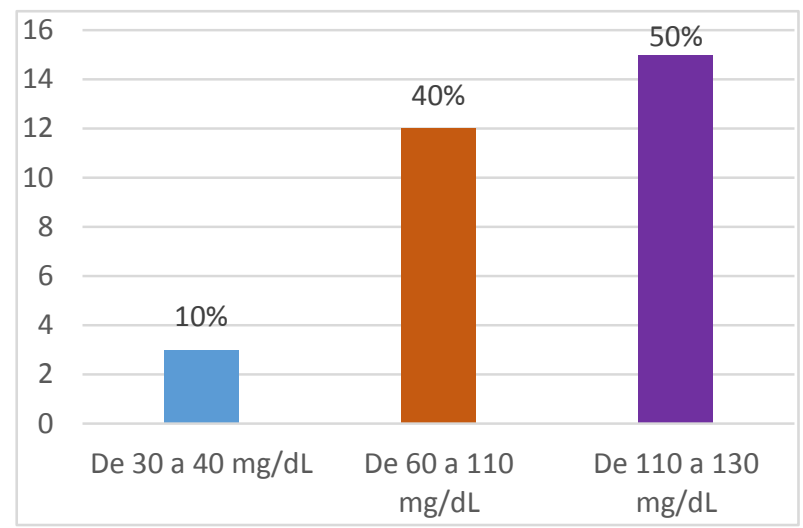

Se observa en la figura 2 que el $10 \%$ de los pacientes tienen el conocimiento de que los valores de Glucosa normales son de 30 a $40 \mathrm{mg} / \mathrm{dl}$ y otro $50 \%$ de 110 a 130 $\mathrm{mg} / \mathrm{dl}$, lo cual es erróneo, se muestra que solo el $40 \%$ de estos pacientes tiene el conocimiento acertado de los valores normales de glucosa de 60 a $110 \mathrm{mg} / \mathrm{dl}$.

Figura 3. Conocimiento sobre niveles altos de glucosa

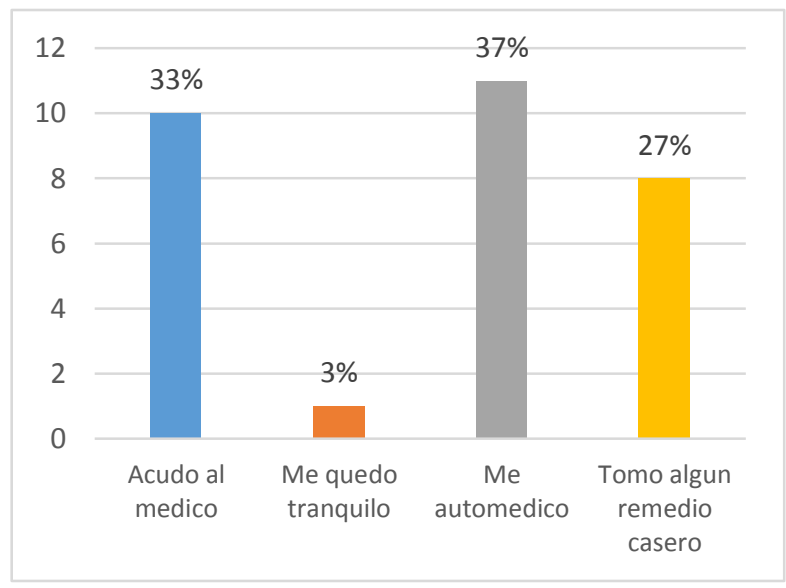

En la figura 3 se muestra que el $37 \%$ de los pacientes se auto medica cuando tienen niveles altos de glucosa, el $27 \%$ decide tomar algún remedio casero en estos casos, el $3 \%$ se queda tranquilo ante esta situación y tan solo el $33 \%$ de estos pacientes toman la decisión de acudir al médico, lo cual hace referencia que el mayor número de pacientes no sabe qué hacer o cómo actuar ante la situación. 
Figura 4. Conocimiento de las complicaciones que pueden generarse debido a la diabetes, indique cuales ha sufrido.

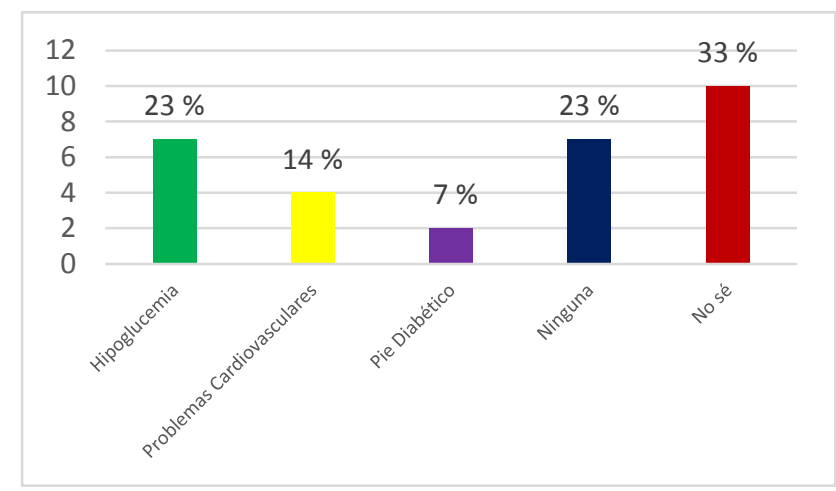

De acuerdo a los datos obtenidos, se muestra en la figura 4 que una tercera parte de los pacientes (33\%) no conoce cuáles son las complicaciones que pueden generarse debido a la diabetes, un $23 \%$ opina que ninguna, otro $23 \%$ opina que puede generarse hipoglucemia, un $14 \%$ dice que problemas cardiovasculares y por ultimo un $4 \%$ de los pacientes dice que pie diabético.

Figura 5 ¿El glucómetro con el que cuenta es?

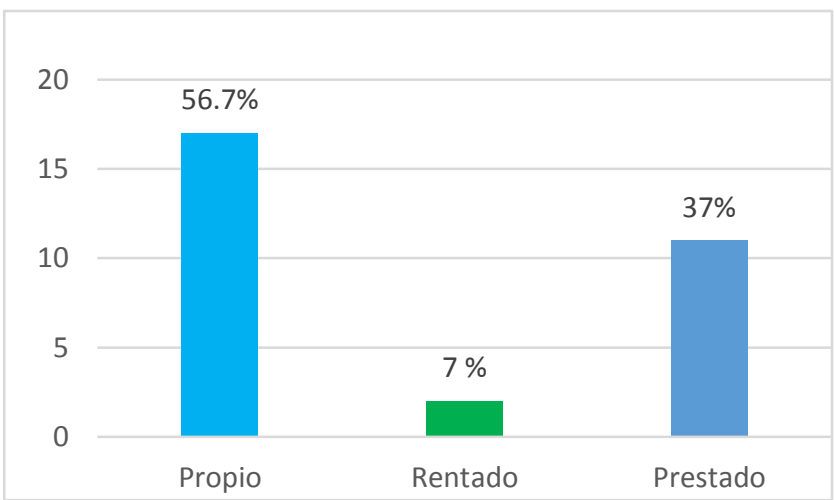

En la figura 5 se observa que del total de los 30 pacientes encuestados, más de la mitad (56\%) cuenta con un glucómetro propio para realizar la técnica de glicemia capilar, a lo que el $7 \%$ respondió que lo renta y el resto $37 \%$ es prestado.
Figura 6. ¿Qué parte de la yema del dedo utiliza para la punción?

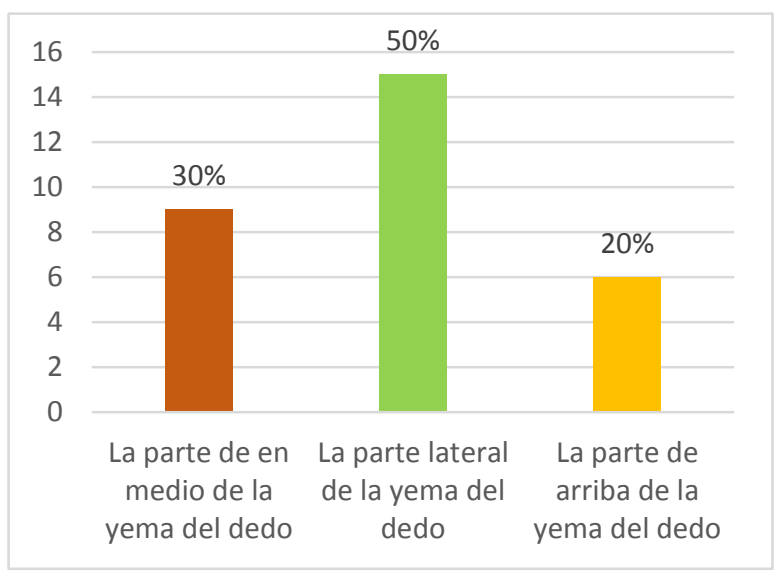

De los 30 pacientes encuestados 9 utilizaban la parte de en medio del dedo para la punción, lo que supone que el $30 \%$ de todos los pacientes. En cuanto a la parte lateral de la yema del dedo la utilizaba el $50 \%$ del total, lo que corresponde a 15 pacientes. Y por último había 6 pacientes que utilizaban la parte de arriba de la yema del dedo, es decir el $20 \%$.

\section{Discusión}

El estudio tiene como propósito analizar el nivel de conocimiento en la técnica del auto monitoreo de glucosa a adultos mayores con diabetes mellitus tipo 2, dado que se considera un problema de salud pública en el ámbito mundial y en México al igual que otros países no escapan de esta problemática.

La prevalencia en la población mexicana es de 4 millones de personas referidas con diagnóstico de diabetes tipo 2 este es el tipo más común de la diabetes, la cual es la primera causa de mortalidad general en México, esta aumenta el riesgo de enfermedades cardiovasculares ${ }^{[12]}$.

Es necesario que las personas tengan conocimiento sobre el manejo de la técnica de auto monitoreo. Se observa en un estudio de González ${ }^{[13]}$ tiene como propósito identificar el nivel de conocimiento de los pacientes en relación con algunos aspectos de la 
educación diabeto lógica encontrando que predomina el género femenino, abunda el grupo de mayores de 65 años, esto coincide con los resultados obtenidos de Barcelo $^{[14]}$, con similitud a este estudio a diferencia de Guzmán $^{[15]}$, prevaleció el sexo masculino el cual tuvo como objetivo explorar el nivel de conocimientos y autocuidado e identificar los niveles de glicemia inicial y final de un grupo de pacientes diabéticos tipo 2 que asisten a sesiones educativas.

De acuerdo al grado de escolaridad más veces señalado es el nivel básico (primaria) de acuerdo al estudio de Guzmán ${ }^{[15]}$ que tiene similitud con este estudio, a lo contrario del estudio de Domínguez ${ }^{[16]}$ en el cual prevalece el nivel de secundaria y preuniversitaria, donde su objetivo es determinar el nivel de conocimientos y utilización del glucómetro en el auto monitoreo de glucosa en personas con diabetes.

De acuerdo al estudio de Evaluación del Programa de Automonitoreo de la Glucosa Capilar de Augusto, et al ${ }^{(17]}$ que tiene por objetivo evaluar estructura, proceso y resultado del programa de automonitoreo de la glucosa capilar en el Municipio Brasileño, donde 9 de 288 que corresponde al $3 \%$ de los pacientes tienen conocimiento acertado acerca de los valores normales de glucosa a diferencia de este estudio donde 12 de 30 pacientes que corresponde al $40 \%$ tienen el conocimiento correcto de los valores de glucosa. Los niveles adecuados de la glucosa en sangre para personas con diabetes recomendados por la Asociación Americana de la Diabetes son: Glucosa plasmática prepandial (antes de comer) 80-130 mg/dl, Glucosa plasmática posprandial (1-2 horas horas después del inicio de la comida) menos de $180 \mathrm{mg} / \mathrm{dl}$. (ADA) ${ }^{(18](19]}$.

Relacionado con la codificación del glucómetro, el estudio de Evaluación de satisfacción del adulto mayor diabético con el uso del glucómetro de Casanova, et al., ${ }^{(20)}$ refiere que la mayor parte de los pacientes de su investigación se les hace más fácil no codificar el glucómetro o no tienen el conocimiento al respecto, relacionado con este estudio donde prevalece con mayor frecuencia que los pacientes no tienen conocimiento sobre la codificación del glucómetro.

Algunos pacientes con diabetes no dimensionan la importancia de codificar con exactitud sus glucómetros y esto los pone en riesgo de calcular mal sus dosis de insulina; en algunas ocasiones, utilizan tiras reactivas caducadas, o no calibran bien el glucómetro para las tiras que están utilizando, esto se debe a que la codificación manual consiste en programar determinados valores a través de una tira codificada o un código para el buen funcionamiento de las tiras; omitir este paso, puede ocasionar que el glucómetro de resultados imprecisos, los cuales originan un tratamiento equivocado o bien se puede contribuir a desarrollar problemas de salud a largo plazo.

En relación con el estudio de Domínguez ${ }^{(16]}$. "Conocimiento y utilización del glucómetro en el automonitoreo de glucosa en personas con Diabetes Mellitus tipo 2" da como resultado que algunas de las personas llegan a tener un nivel de conocimiento relacionado con su enfermedad, pero más de la mitad de las personas su conocimiento es deficiente en gran porcentaje, relacionado con este estudio la prevalencia de personas que tienen un conocimiento con respecto a la Diabetes Mellitus está muy por debajo de las personas que llegan a tener noción de su enfermedad.

\section{Conclusión}

La diabetes mellitus se considera un problema de salud pública en el ámbito mundial y en México, es por ello por lo que se decidió investigar sobre el conocimiento que llega a tener un paciente conforme al automonitoreo de la glucemia capilar.

En este estudio, de acuerdo con los resultados obtenidos de la muestra, predominò el sexo femenino con ocupación de Ama de casa, en donde el mayor rango de edad fue de 62 y 68 años, con un mayor grado de escolaridad nivel básico. 
En los resultados se logró identificar que toda la muestra cuenta con glucómetro propio, basándonos en el nivel de conocimiento, se observó que más de la mitad realiza su automonitoreo con un horario de la mañana y que únicamente lo realizan una vez al día, en donde se explica que realizan lavado de manos antes del automonitoreo en la parte lateral de la yema del dedo, al igual que la mayor parte desinfecta la zona de punción con alcohol, pero lo realizan Rara vez. En cuanto a la codificación del glucómetro no saben realizarla o realmente no saben lo que significa, al igual que no saben cómo o cuando calibrar su glucómetro, referente a la limpieza del glucómetro, poco más de la muestra suele limpiar su glucómetro cada mes y suele utilizar una nueva lanceta por más de cinco ocasiones durante su automonitoreo.

\section{Referencias}

[1]. Organización Mundial de la Salud. Informe mundial sobre la diabetes. [en línea]. [citado 22 enero de 2019]; Recuperado en: https://www.who.int/diabetes/global-report/es/ .

[2]. Castro Meza, A., \& Salcedo Álvarez, R. La enseñanza a pacientes con diabetes. Enfermería Universitaria, 14(1) 39- 46. 2017

[3]. Dennis Kasper, A. F. Harrison. Principios de medicina interna México, D.F.: McGraw-Hill Interamericana Editores, S.A. de C.V. (2016).

[4]. Priscilla LeMone, K. B. Enfermería medico quirúrgica. Pensamiento crítico en la asistencia del paciente. Madrid (España): Pearson Educación S. A. (2009)

[5]. Schaurich D, Crossetti Olivera MG. Produçãodo conhecimento sobre teorías de enfermagem: análise de periódicos da área, 1998-2007. Esc Anna Nery [en linea ]. 2010 [citado 9 Jul 2018];14(1):182-88. Recuperado

http://www.scielo.br/scielo.php?script=sci_arttext\&pid=S1414-

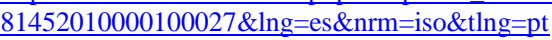

[6]. Pereda-Acosta M. Explorando la teoría general de enfermería de Orem. Enf Neurol (Mex) [en linea]. 2011[citado 9 Jul 2018];10(3):163 67. Recuperado en: http://new.medigraphic.com/cgibin/contenido.cgi?IDREVISTA=221\&IDPUBLICACION=3438

[7]. Marcos-Espino MP, Tizón-Bouza E. Aplicación del modelo de Dorothea Orem ante un caso de una persona con dolor neoplásico. Gerokomos [en linea]. 2013 [citado 9 Jul 2018]; 24(4). Recuperado en: http://dx.doi.org/10.4321/S1134-928X2013000400005
[8]. Raimondo ML, Fegadoli D, MéierMarineli J, Wall Marilene L, Labronici LM, Raimondo-Ferraz MI. Produção científica brasileira fundamentada na Teoria de Enfermagem de Orem: revisão integrativa. Rev Bras. Enferm [en linea]. 2012 [citado 9 Jun 2018]; 65(3):529-34. Recuperado

http://www.scielo.br/scielo.php?script=sci_arttext\&pid=S0034 $71672012000300020 \& \operatorname{lng}=$ en $\& n r m=$ iso\&tlng=en

[9].Vidal M. Monitorización glucémica y educación terapéutica en la diabetes. [en linea]. 2010 [citado 9 mayo 2019]; 26(Supl 1):15-28. Recuperado https://es.scribd.com/document/235962057/Monitorizacion-Glucemica-yEducacion-Terapeutica-en-La-Diabetes

[10]. Guía de practica clinica para el diagnostico, tratamiento y seguimiento de la diabetes mellitus tipo 2 en la poblacion mayor de 18 años. [en linea]. 2016 [citado 15 mayo 2019]. Recuperado en: http://gpc.minsalud.gov.co/gpc_sites/Repositorio/Conv_637/GPC_diabete s/DIABETES_TIPO_2_COMPLETA.pdf

[11].FDA. Como usar de manera segura los medidores de glucosa y las tiras reactivas para la diabetes. [en línea]. 2019 [citado 20 marzo de 2019]; Recuperado en: https://www.fda.gov/ForConsumers/ConsumerUpdates/.../ucm558745.htm

[12]. . Federacion Mexicana de Diabtes La diabetes en Mexico, [en línea]. 2014 [citado 21 mayo de 2019]; Recuperado en: http://fmdiabetes.org/wpcontent/uploads/2018/04/La diabetes_en_Me\%CC\%81xico.jpg

[13].Gonzalez-Marante CA, Bandera-Chapman S, Valle-Alonso J, Fernandez-Quesada J. Conocimientos del diabetico tipo 2 acerca de su enfermedad. MED GEN Y FAM. [en línea] 2015 [citado 21 mayo de 2019]; 4(1):10-15. Recuperado en:

https://doi.org/10.1016/j.mgyf.2015.05.003

[14]. Barcelo A Estudio de la prevalencia de diabetes mellitus en un área rural de salud. Rev. Cubana Med Gen Integr., 34 (2003) 109.115

[15].Guzmán-Pérez MI, Cruz-Cauich AJ, Parra-Jiménez J, ManzanoOsorio M. Control glicémico. Conocimientos y autocuidado de pacientes diabéticos tipo 2 que asisten a sesiones educativas. Rev Enferm IMSS 2005; 13(1): 9-13

[16].Domínguez Y, Álvarez-Vázquez I, González-Calero T, Conesa González A, Cruz- Hernández J. Conocimientos y utilización del glucómetro en el automonitoreo de glucosa en personas con diabetes. [en línea] 2018 [citado 21 mayo de 2019]; 18 (4):1693-1699. Recuperado en:

http://www.saludpublica.es/secciones/revista/revistaspdf/bc5c02c0923362 5_Hig.Sanid.Ambient.18.(4).1693-1699.(2018).pdf

[17]. Augusto MC, Trevizani MJ, Garcia de Lima CM, Zanetti ML, de Barros MA, Carvalhaes L Evaluación del Programa de Automonitoreo de la Glucosa Capilar. [en línea] 2018 [citado 21 mayo de 2019]; 22 (5):8019. Recuperado en: www.scielo.br/pdf/rlae/v22n5/es_0104-1169-rlae-2205-00801.pdf 
Publicación semestral, Educación y Salud Boletín Científico Instituto de Ciencias de la Salud Universidad Autónoma del Estado de Hidalgo, Vol. 8, No. 14 (2019) 197-205

[18]. American Diabetes Association. Standars of medical care in diabetes: Official Web site of the American Diabetes Association [en línea] 2017 [citado 29 mayo de 2019]. Recuperado en www. https://professional.diabetes.org/files/media/dc_40_s1_final.pdf

[19] American Diabetes Association. Control de la glucosa

Official Web site of the American Diabetes Association

[en línea] 2015 [citado 29 mayo de 2019]. Recuperado en:

.http://www.diabetes.org/es/vivir-con-diabetes/tratamiento-y-cuidado/elcontrol-de-la-glucosa-en-la-sangre/control-de-la-glucosa.html

[20].Casanova MC, Ramos D, Trasancos M, Ochoa MR, García CA, Hernández F. Evaluaciòn de satisfacciòn del adulto mayor diabètico con el uso de glucòmetro. [en línea] 2016 [citado 18 mayo 2019]; 20 (4):801-9. Recuperado en:

http://scielo.sld.cu/scielo.php?script=sci_arttext\&pid=S1025$\underline{02552016000400010}$ 